\title{
MicroRNA-451 suppresses tumor cell growth by down-regulating IL6R gene expression ${ }^{\text {th }}$
}

\author{
Dong Liu ${ }^{\mathrm{a}}$, Cong Liu ${ }^{\mathrm{b}}$, Xiyin Wang ${ }^{\mathrm{b}}$, Sigurdur Ingvarsson ${ }^{\mathrm{c}}$, Huiping Chen ${ }^{\mathrm{b}, *}$ \\ a Department of Radiology, Tongji Hospital, Tongji Medical College, Huazhong University of Science and Technology, Wuhan, Hubei, China \\ ${ }^{\mathrm{b}}$ Department of Medical Genetics, Tongji Medical College, Huazhong University of Science and Technology, Wuhan, Hubei, China \\ ' Institute for Experimental Pathology and Faculty of Medicine, University of Iceland, Keldur, Reykjavik, Iceland
}

\section{A R T I C L E I N F O}

\section{Article history:}

Received 17 September 2013

Received in revised form 15 December 2013

Accepted 19 December 2013

Available online 17 January 2014

\section{Keywords:}

miR-451

Tumor suppressor

IL6R

Down-regulating

\begin{abstract}
A B S T R A C T
The miR-451 was found to be frequently down-regulated in tumors, indicating that miR-451 could play an important role in carcinogenesis. This study uncovered the mechanism by which the miR-451 functions as a tumor suppressor. The target genes of miR-451 were determined using target gene prediction softwares. Then the miR-451 mimics were introduced into RKO and Hela cells respectively. The proliferation and invasion of cells were monitored by MTT, cell cycle and in vitro extracellular matrix invasion assays. Also the angiogenesis of HUVEC cells transfected with miR-451 mimics was examined. Subsequently, IL6R, a predicted target gene of miR-451, was studied by real time PCR, Western blotting, and siRNA technologies. The mRNA and protein levels of IL6R gene were found to be down-regulated in the RKO and Hela cells transfected with miR-451 mimics. Consequently, the cell proliferation was inhibited. Also, the invasion of RKO cells was suppressed. Furthermore, the angiogenesis of HUVEC cells transfected with miR-451 mimics was assayed and the decreased angiogenic ability was detected compared to the controls. All these results were validated by IL6R siRNA experiments. The IL6R gene is a target gene of miR-451. The miR-451 behaves as a tumor suppressor, probably by targeting the IL6R pathway.
\end{abstract}

(c) 2013 Elsevier Ltd. All rights reserved.

\section{Introduction}

Most oncogenes and tumor-suppressor genes are first transcribed from DNA into RNA, and then translated into proteins to exert their effects. Increasing evidence indicates that small noncoding RNA molecules, called microRNA (miRNAs), can also function as tumor suppressors and oncogenes [1]. The miRNAs are short, endogenous, non-coding RNAs known to inhibit the translation of their target transcripts and have wide range of roles in differentiation, development, growth and apoptosis [2]. In mammals, miRNAs are transcribed by RNA polymerase II as primary-miRNA (pri-miRNA) [3]. The pri-miRNA is cleaved by the Drosha ribonuclease III enzyme to generate an approximately 70nt stem-loop precursor miRNA (pre-miRNA), which is exported into the cytoplasm by the transporter exportin 5 [4]. Subsequently,

\footnotetext{
This project was supported by the Huazhong University of Science and Technology (2010MS034 and HF-09-37-2011-510).

* Corresponding author at: Department of Medical Genetics, Tongji Medical College, Huazhong University of Science and Technology, Hangkong Lu 13, Wuhan, Hubei 430030, China. Tel.: +86 27 83692629; fax: +86 2783692628 .

E-mail addresses: huiping@mail.hust.edu.cn, huiping65@hotmail.com (H. Chen).
}

it is further cleaved by the Dicer ribonuclease to produce the mature miRNA and antisense miRNA star (miRNA*) products [5]. The mature miRNA is incorporated into a RNA-induced silencing complex (RISC), which most commonly results in translational inhibition or destabilization of the target mRNAs [6,7].

In cells, miRNAs regulate gene expression in a specific and effective manner. This regulation is considered to be economic and energy saving. Consequently, miRNAs is involved in many processes in cells. The miRNA-451 (miR-451) gene is located at 17q11.2. It has been found to be expressed in many human tissues, and showed a high expression level in fetuses compared to adults [8-10]. Specially, the miR-451 has 5 variants, indicating that it could play a major role in cell growth and differentiation [9]. However, miR-451 exhibited a lower expression level in several cancers [11-13], suggesting that it may be engaged in the carcinogenesis. It is of interest to understand how the miR-451 functions in tissue cells or cancers, namely what its target genes are. To date only few studies have reported on the mechanism of miR-451 in gene expression regulation.

In the present study, we investigated relationship between miR-451 and its candidate target gene IL6R in human colon carcinoma cell line RKO, human cervical cancer cell line Hela and human umbilical vein endothelial cells (HUVEC). After transfecting 
miR-451 mimics, we quantified IL6R mRNA expression and investigated the expression of the IL6R proteins in RKO and Hela cells. Taking together, we found that transfection of miR-451 down-regulated IL6R expression at both mRNA and protein level. Finally, we found that the transfection of miR-451 suppressed cell proliferation and invasion and angiogenesis. These findings gave new insights into the roles of miR-451 in tumourigenesis.

\section{Materials and methods}

\subsection{Target gene prediction}

Mature miR-451 sequence was obtained from miRBase (http:// www.mirbase.org/). Five web-based miRNA target prediction programs were used for the prediction of miR-451 potential target genes: TargetScans (http://www.targetscan.org/index.html), DIANA LAB (http://diana.cslab.ece.ntua.gr/microT/), microRNA.org (http:// www.microrna.org/microrna/home.do), PicTar (http://pictar.mdcberlin.de/), Microcosm Targets (http://www.ebi.ac.uk/enright-srv/ microcosm/cgi-bin/targets/v5/search.pl). The candidate target genes were chosen based on high miRNA:mRNA alignment scores $[14,15]$.

\subsection{Cell lines and cell culture}

The RKO, Hela and HUVEC cell lines were purchased from Chinese Academy of Sciences (China). The RKO and Hela cells were maintained in MEM medium (Hyclone, USA) supplemented with $10 \%$ fetal bovine serum (FBS) (Hyclone, USA), $1 \mathrm{mM}$ sodium pyruvate (Hyclone, USA), 1.5\% sodium bicarbonate (Hyclone, USA) and 100 units/ml penicillin/streptomycin (Invitrogen, USA). The HUVEC cells were cultured in RMPI 1640 (Hyclone, USA) supplemented with 10\% FBS (Hyclone, USA) and 100 units/ml penicillin/streptomycin (Invitrogen, USA). These cells were incubated at $37{ }^{\circ} \mathrm{C}$ in a humidified chamber containing $5 \% \mathrm{CO}_{2}$ atmosphere. It was routinely passaged at 1-2 day intervals.

\subsection{Oligonucleotide synthesis and transfection}

The mimics for miR-451 wildtype and variant, and IL6R-siRNA were chemically synthesized by Ruibo company (China). Their sequences were shown in Table 1. The RKO, Hela and HUVEC cells were seeded into each well of 6-well plates respectively, and incubated in antibiotic-free medium overnight. The oligonucleotides above were then transfected into RKO and Hela cells respectively using Lipofectamine 2000 reagent (Invitrogen, USA) at the time of $40-50 \%$ confluence. According to the manufacturer's instructions, the transfection complexes containing $100 \mathrm{nmol}$ of oligonucleotides were added directly to the cells. At 4-6 h after transfection, the medium was replaced with fresh MEM or RPMI1640 containing 10\% FBS, and the cells were incubated for an additional $48 \mathrm{~h}$ and then harvested.

\subsection{RNA isolation and PolyA reverse transcription}

The small RNAs ( $\leq 200 \mathrm{nt}$ ) were isolated from the RKO and Hela using a mirVanaTM miRNA isolation kit (Ambion, USA) following the manufacturer's instructions. The small RNAs were eluted in $100 \mu$ l RNase-free water. The RNA concentration was tested by UV absorbance at $260 \mathrm{~nm}$. The small RNAs were polyadenylated at $37^{\circ} \mathrm{C}$ for $30 \mathrm{~min}$ in $50 \mu \mathrm{l}$ reaction volume using $5 \mu \mathrm{g}$ RNA and $5 \mathrm{U}$ poly(A) polymerase (New England Biolabs, USA). Then the poly(A)tailed small RNAs were purified through phenol/chloroform extraction and ethanol precipitation. Reverse transcription was conducted using the entire poly(A)-tailed RNA and $10 \mu \mathrm{M}$ RT primer (5'-CGC TAC GTA ACG GCA TGA CAG TG(T)24-3') (Table 1) with $200 \mathrm{U}$ of SuperScript III reverse transcriptase (Invitrogen, USA) according to the manufacturer's instructions.

\subsection{Assessment of the endogenous and transfected miRNAs by quantitative PCR}

Real-time PCR was carried out in a ABI StepOnePlus system with the miRNA-specific forward primers (5'-AAA CCG TTA CCA TTA CTG AGT T- $3^{\prime}$ ) and reverse primer (5'-CGC TAC GTA ACG GCA TGA CAG TG-3') (Table 1), and TransStart SYBR green qPCR supermix (TransGen, Biotech) following the manufacturer's instructions. The $20 \mu \mathrm{l}$ reaction mixture including $0.5 \mu \mathrm{l}$ of cDNA synthesized above, $10 \mu \mathrm{l}$ TransStart SYBRgreen qPCR supermix and $0.5 \mathrm{mM}$ forward and reverse primers was incubated at $95{ }^{\circ} \mathrm{C}$ for $5 \mathrm{~min}$, followed by 40 cycles of $95{ }^{\circ} \mathrm{C}$ for $10 \mathrm{~s}, 55^{\circ} \mathrm{C}$ for $15 \mathrm{~s}$ and $72{ }^{\circ} \mathrm{C}$ for $20 \mathrm{~s}$. Melting curves for each PCR were carefully monitored to avoid non-specific amplification. The U6 small nuclear RNA was used as the internal control. The primers used were shown in Table 1. Each sample was analyzed in triplicate. The relative level (RL) of each miRNA expression was calculated with $2^{-\Delta \Delta \mathrm{Ct}}$ method.

\subsection{Analysis of IL6R mRNA by quantitative RT-PCR}

Total RNAs were isolated from RKO and Hela using TRIZol ${ }^{\circledR}$ Reagent (Invitrogen, USA) following the manufacturer's instructions, and the total RNAs were eluted in $50 \mu$ l RNAse-free water. The RNA concentration was tested by UV absorbance at $260 \mathrm{~nm}$. The total RNAs were reverse transcribed using RevertAid First Strand cDNA Synthesis Kit (Thermo Scientific, USA) following the manufacturer's instructions. Real-time PCR was carried out in an

Table 1

The oligonucleotide sequences used in the study.

\begin{tabular}{|c|c|}
\hline Oligonucleotide & Sequence \\
\hline Forward primer for IL6R RT-PCR & CTC CTG CCA GTT AGC AGT CC \\
\hline Reverse primer for Il6R RT-PCR & TCT TGC CAG GTG ACA CTG AG \\
\hline Forward primer for GAPDH RT-PCR & GAG TCA ACG GAT TTG GTC GTC \\
\hline Reverse primer for GAPDH RT-PCR & TTG ATT TTG GAG GGA TCT CG \\
\hline Forward primer for miR-451 RT-PCR & AAA CCG TTA CCA TTA CTG AGT T \\
\hline Reverse primer for miR-451 RT-PCR & CGC TAC GTA ACG GCA TGA CAG TG \\
\hline U6 forward primer for RT-PCR & CGC TTC GGC AGC ACA TAT AC \\
\hline U6 reverse primer for RT-PCR & AAA ATA TGG AAC GCT TCA CGA \\
\hline miR-451 wildtype mimic & AAA CCG UUA CCA UUA CUG AGU U \\
\hline miR-451 variant mimic & ACA CCG UUA CCA UUA CUG AGU UU \\
\hline IL6R-siRNA (si-IL6R) & CCA CGA CUC UGG AAA CUA U dTdT \\
\hline Negative control miRNA (NTC M) & UUU GUA CUA CAC AAA AGU ACU G \\
\hline Negative control siRNA (NTC S) & UUC UCC GAA CGU GUC ACG UUC UG \\
\hline RT primer & CGC TAC GTA ACG GCA TGA CAG TG(T)24 \\
\hline
\end{tabular}


ABI StepOnePlus system with the IL6R-specific forward primer (5'CTC CTG CCA GTT AGC AGT CC-3') and reverse primer (5'-TCT TGC CAG GTG ACA CTG AG-3') (Table 1), and TransStart SYBR green qPCR supermix (TransGen, China) following the manufacturer's instructions. The $20 \mu \mathrm{l}$ reaction mixture including $0.5 \mu \mathrm{l}$ of cDNA, $10 \mu \mathrm{l}$ TransStart SYBRgreen qPCR supermix and $0.5 \mathrm{mM}$ forward and reverse primers was incubated at $95^{\circ} \mathrm{C}$ for $5 \mathrm{~min}$, followed by 40 cycles of $95{ }^{\circ} \mathrm{C}$ for $10 \mathrm{~s}, 55^{\circ} \mathrm{C}$ for $15 \mathrm{~s}$ and $72{ }^{\circ} \mathrm{C}$ for $20 \mathrm{~s}$. Melting curves for each PCR were carefully monitored to avoid non-specific amplification. The expression level of IL6R mRNA was normalized by glyceraldehyde-3-phosphate dehydrogenase (GAPDH) expression with $2^{-\Delta \Delta \mathrm{Ct}}$ method. The primers for amplifying GAPDH were listed in Table 1. Each sample was analyzed in triplicate.

\subsection{Western blotting analysis}

The harvested RKO and Hela cells were lysed in RIPA lysis buffer with protease inhibitors (1 $\mathrm{mM}$ PMSF) to isolate proteins, which were quantified using the BCA protein assay (Aidlab, China). Equal amounts of protein samples $(\sim 40 \mu \mathrm{g})$ were separated on a $12 \%$ SDS-PAGE and electrophoretically transferred onto PVDF membrane (Millipore, USA). After blocking with $5 \%$ non-fat milk in TBSTween-20 for $5 \mathrm{~h}$ at room temperature, the membranes were blotted with the appropriate primary IL6R antibody (GeneTex, USA) at a 1:500 dilution overnight at $4{ }^{\circ} \mathrm{C}$. After TBST washing, the membranes were incubated with the secondary anti-rabbit antibody linked to horseradish peroxidase at a 1:5000 dilution for $1.5 \mathrm{~h}$ at room temperature. The protein expression level was normalized with GAPDH (Abcam, USA). After TBST washing, the specific binding antibody was detected using the enhanced chemiluminescent assay (BeyoECLPlus, China) according to the manufacturer's protocol. Each sample was assayed in three independent experiments. The intensity of the bands was examined by using QUANTITY ONE 4.62 (Bio-Rad, USA).

\subsection{Cell proliferation MTT assay}

The RKO and Hela cells were seeded into 96 well plates at a density of $5.0 \times 10^{3}$ cells per well with six replicates, respectively. At 24, 48, and $72 \mathrm{~h}$ after transfection, $10 \mu \mathrm{l}$ MTT (Sigma, USA) reagent was added to each well, and the mixture was incubated for $4 \mathrm{~h}$. After the incubation, the supernatant was removed and $100 \mu \mathrm{l}$ dimethl sulfoxide (DMSO) was added to dissolve the formazan crystals. Optical density (OD) value of each sample was measured at a wavelength of $490 \mathrm{~nm}$ on a Microplate Reader (UNRISE TECAN, Switzerland). The cell proliferation rate was measured by the following formula: $(A M-A C) / A C \times 100 \%$, where $A C$ means absorbance value of the negative control groups and AM means absorbance value of the experimental groups.

\subsection{Cell cycle assay by flow cytometry}

The RNA oligonucleotides were transfected to RKO cells grown in 6-well plate for flow cytometry assay. At $24 \mathrm{~h}$ after transfection, the cells were re-plated into three wells. Then, additional $24 \mathrm{~h}$ later the cells were harvested for flow cytometry assay. Cells were washed with phosphate-buffered saline (PBS) before fixation in ethanol. The fixed cells were treated with $20 \mu \mathrm{g} / \mathrm{ml}$ DNase-free RNaseA in PBS at $37^{\circ} \mathrm{C}$ for $30 \mathrm{~min}$, stained with propidium iodide (PI) at room temperature for $5 \mathrm{~min}$. The ratio of cells in different stages of cell cycle was analyzed by flow cytometry.

\subsection{In vitro extracellular matrix invasion assays}

The cancer cell invasion in vitro was demonstrated using 24well transwell units (Corning, USA) with a $8 \mu \mathrm{m}$ pore size polycarbonate filter coated with Matrigel ${ }^{\circledR}$ (matrigel basement Membrane USA) to form a continuous thin gel. The RKO Cells $\left(\sim 4 \times 10^{5}\right)$ maintained in serum-free medium containing $0.1 \%$ BSA were added to the upper chamber. The lower chamber contained $500 \mu \mathrm{l}$ MEM medium with 10\% FBS. Cells were incubated for $24 \mathrm{~h}$ at $37{ }^{\circ} \mathrm{C}, 5 \% \mathrm{CO}_{2}$ incubator. Then cells on the upper surface of the filter were completely removed with a cotton swab. The filters were fixed in methanol and stained with Hematoxylin Staining Solution. Cells that invaded the matrigel and reached the lower surface of the filters were counted under microscopy at a magnification of $100 \times$. Each experiment was repeated in triplicate.

\subsection{In vitro angiogenesis assay}

The angiogenic ability was assessed using Matrige ${ }^{\circledR}$ assay and in vitro angiogenesis assay on Matrigel ${ }^{\mathbb{R}}$ culture. The HUVEC cells were transfected as described above. At $24 \mathrm{~h}$ after transfection the HUVEC cells were seeded on Matrigel ${ }^{\circledR}$ pre-coated 96-well plate at a density of $5 \times 10^{4}$ cells per well. The angiogenic ability was assessed at $8 \mathrm{~h}$ after plating on Matrige ${ }^{\circledR}$, through vessels count/ size calculated by Image PRO-Plus 6.0.

\subsection{Statistical analysis}

A two-tailed Student's $t$ test was used to analyze differences between the parameters. $P \leq 0.05$ was regarded as a significant difference.

\section{Results}

\subsection{IL6R gene was chosen to be studied}

Based on high scores of miRNA:mRNA alignment through five software tools, 4 genes were chosen for further analysis: EMSY, IL6R, OSR1, and YTHDF2. After being examined rapidly by RT-PCR and realtime PCR, the IL6R gene was regarded as a potential target gene of miR-451. The $5^{\prime}$ bases $2-7$ of miR-451, the seed region of miRNAs, matches completely with 3' UTR region of IL6R mRNA (Fig. 1A).

\subsection{Low level of endogenous miR-451 and high efficiency of transfection of miRNA mimics}

The expression level of endogenous miR-451 in both RKO and Hela was examined using real-time PCR. A low level of miR451 was detected in both cell lines $(P<0.001$, Fig. $1 B$ and $C)$. The miRNA mimics were transfected into both cell lines, and a high efficiency of transfection was identified (Fig. 1B and C).

\subsection{The IL6R gene was knocked down in both mRNA and protein levels}

The mRNA and protein levels of the IL6R gene were analyzed in the RKO and Hela cells after transfections with miR-451 mimics. A significant inhibition was seen in both mRNA and protein levels $(P<0.001$, Figs. 2 and 3$)$. Interestingly, the miR-451 variant does not suppress IL6R gene even though there is only one base difference in the seed sequence between the wild type and the variant (Table 1).

\subsection{The miR-451 inhibited cell proliferation, invasion and angiogenesis}

The transfected RKO and Hela were tested for cell proliferation rate by MTT experiments. The relative proliferation rates for cells transfected with miR-451 mimics were significantly lower than the controls $(P<0.001$, Fig. 4$)$. Furthermore, the cell cycle assay was performed in the RKO cells. The increased cell percentages at 
A
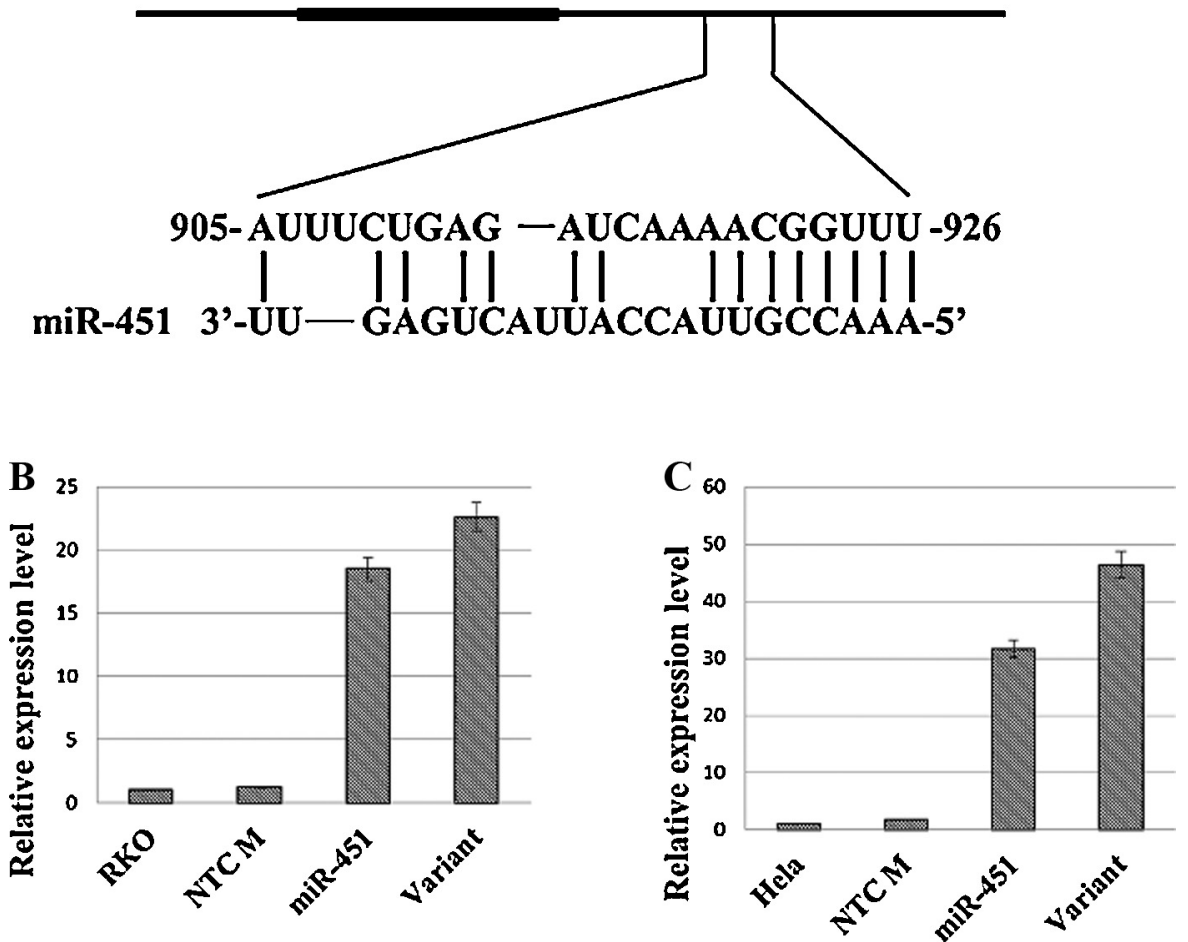

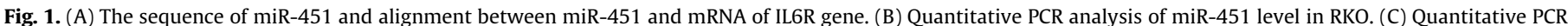

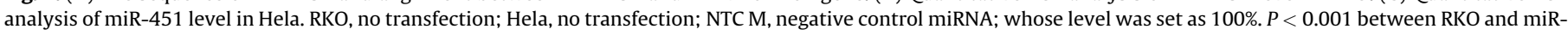

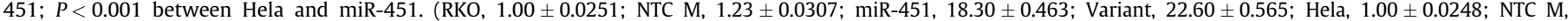
$1.78 \pm 0.0461 ;$ miR-451, $31.60 \pm 0.791$; Variant, $46.30 \pm 1.158$ ).
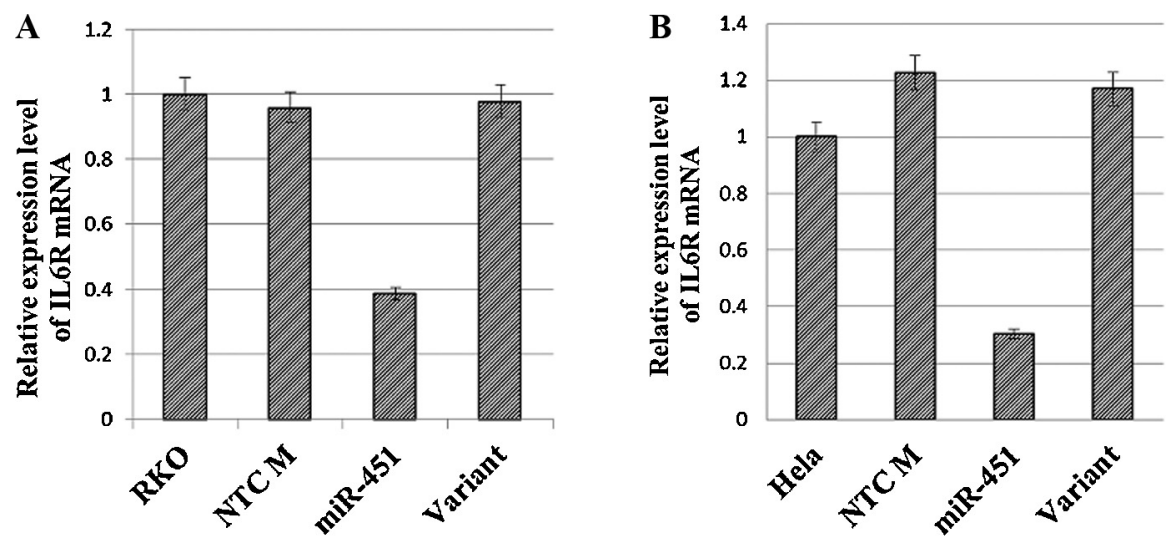

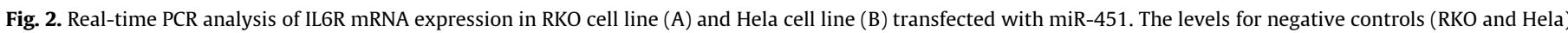

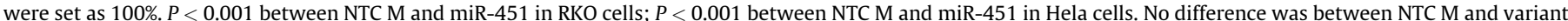

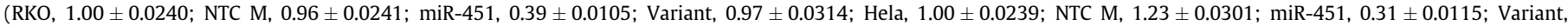
$1.17 \pm 0.0276)$.

G0-G1 phases and decreased cell percentages at S phases were seen for miR-451 mimics compared to controls $(P<0.001$, Fig. 4$)$. The invasive properties of the RKO cell line were assayed using transwell units. The relative invasion values for miR-451 mimics were significantly decreased compared to controls $(P=0.001$, Fig. 5). Moreover, the impact of miR-451 on the angiogenic ability of the HUVEC cells was analyzed. The angiogenic ability for miR451 mimic transfected cells was significantly lower than controls $(P=0.006$, Fig. 6).

\subsection{The siRNA experiments validated results on miRNAs}

The RNA oligonucleotides with homology to the coding region of IL6R gene were transfected into both RKO and Hela cells. The IL6R proteins were then tested by Western blotting, and an obvious inhibition of expression was seen compared to controls (Fig. 7). Consequently the cell cycle and proliferation, invasion and angiogenesis were assayed. The results were in line with those on miRNAs (Figs. 4-6). 

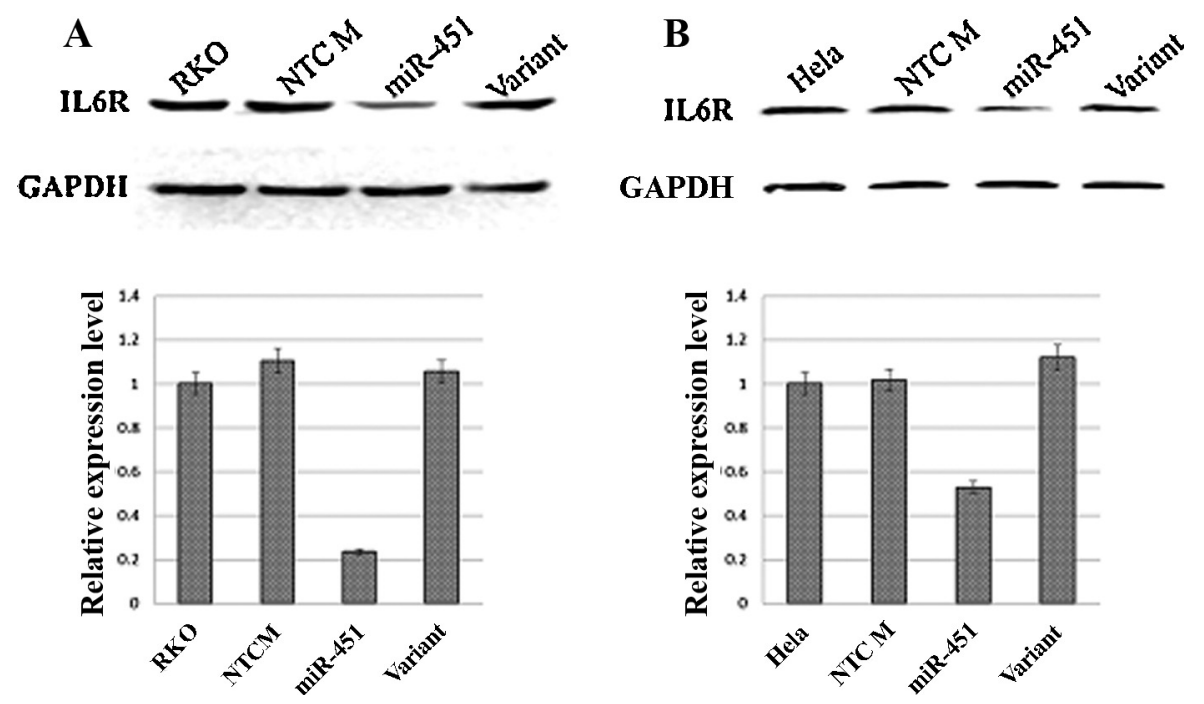

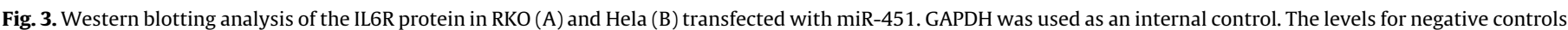

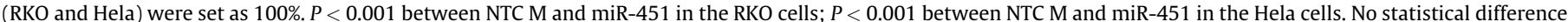

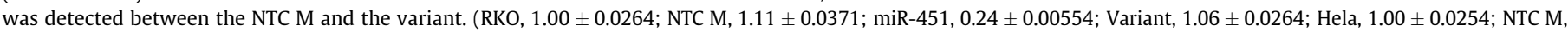
$1.02 \pm 0.0240 ;$ miR-451, $0.53 \pm 0.0132$; Variant, $1.12 \pm 0.0289$ ).
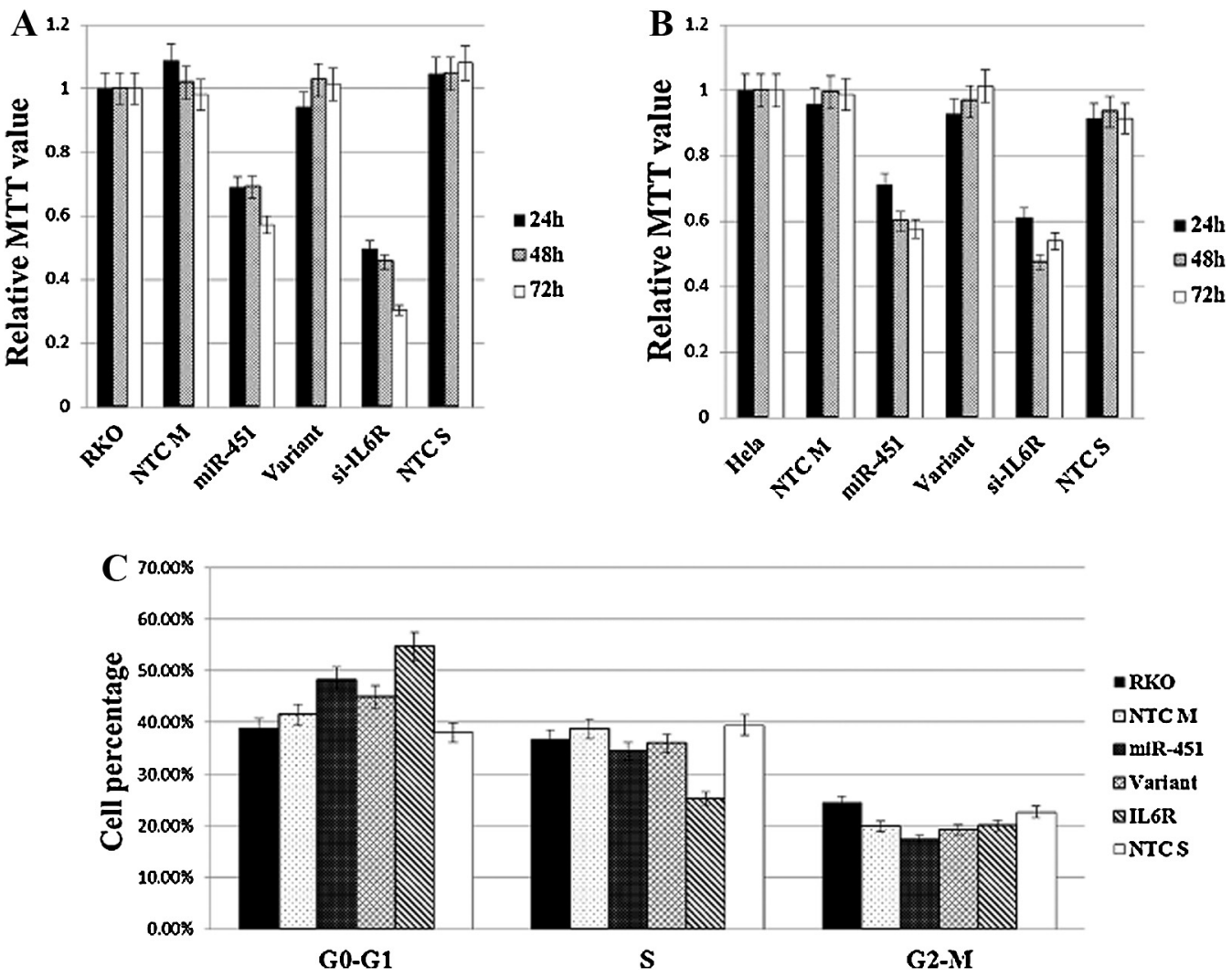

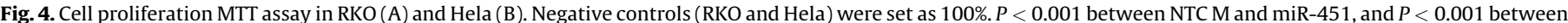

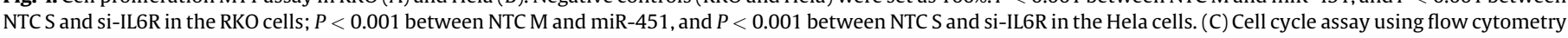

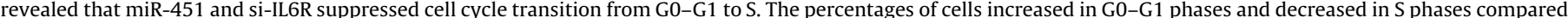

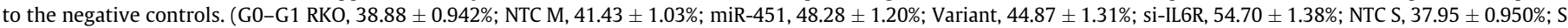

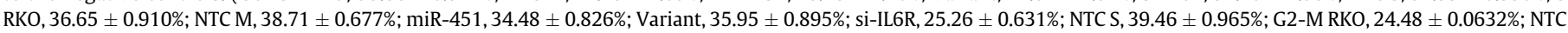
M, $19.86 \pm 0.491 \%$; miR-451, $17.23 \pm 0.475 \%$; Variant, $19.18 \pm 0.495 \%$; si-IL6R, $20.04 \pm 0.541 \%$; NTC S, $22.60 \pm 0.585 \%$ ).

\section{Discussion}

The target genes of miR-451 have been explored by few research groups so far. One article reported that CAB39 is a target gene of miR-451, and that it may regulate the CAB39/AMPK pathway [16]. Another group described that miR-451 downregulation allows the expression of the direct target gene macrophage migration inhibitory factor, which is involved in the expression of COX2. In turn, COX2 allows Wnt activation, which is essential for growth of cancer stem cells. It is conceivable 
A
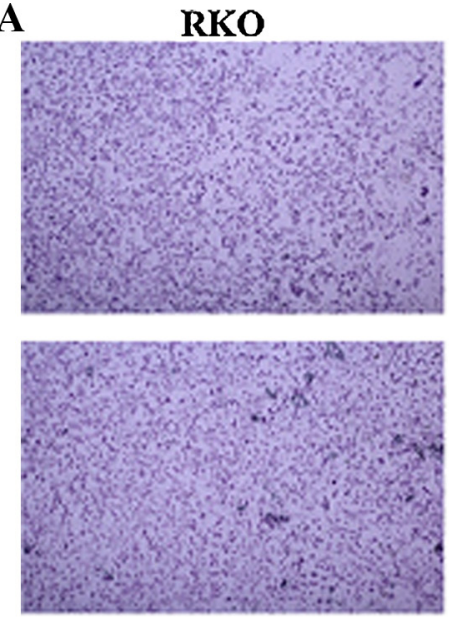
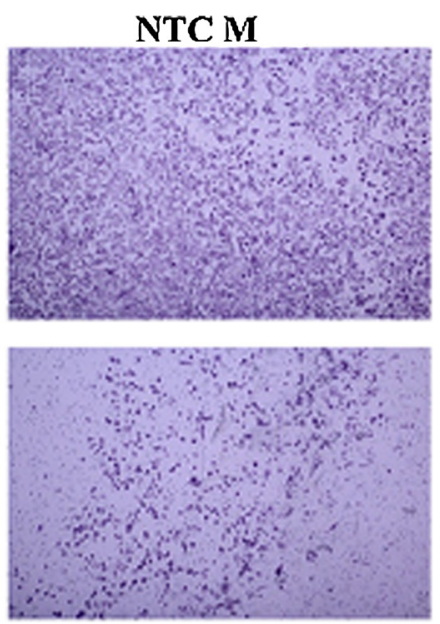
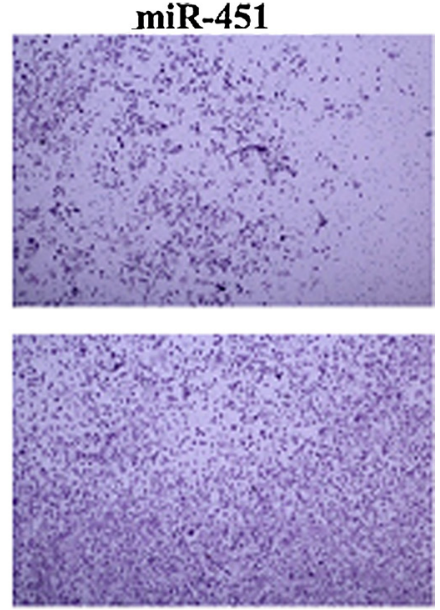

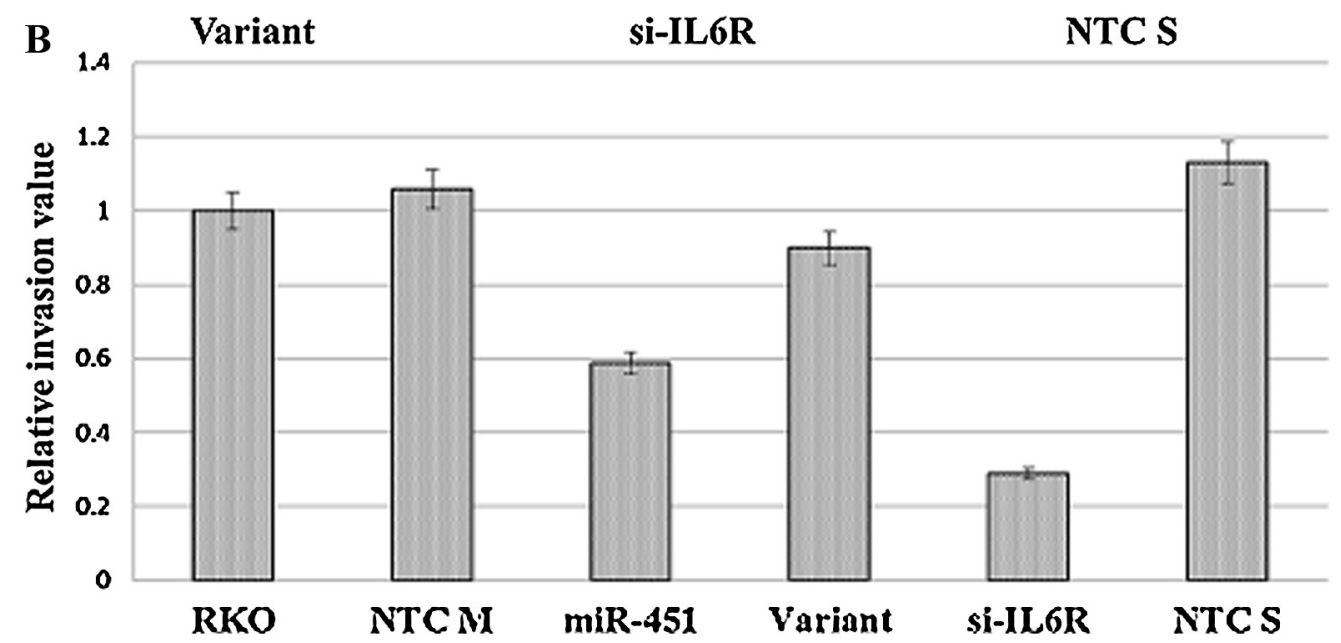

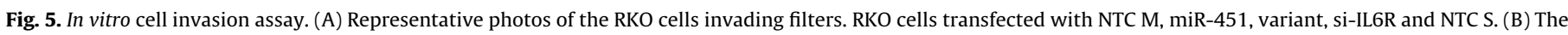

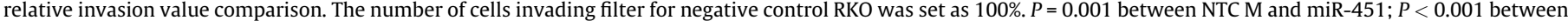
NTC S and si-IL6R. (RKO, $1.00 \pm 0.0204$; NTC M, $1.06 \pm 0.0261 ;$ miR-451, $0.59 \pm 0.0142$; Variant, $0.89 \pm 0.0293 ;$ si-IL6R, $0.29 \pm 0.00728 ;$ NTC S, $1.12 \pm 0.0289$ ).

to expect that there could be additional target genes for miR-451 that have not been detected so far.

In this study, we used software prediction and miR-451 mimic transfection to identify a target gene of miR-451, and found that IL6R is a candidate. The finding was confirmed by siRNA experiments. Introduction of miR-451 mimics into cells generated inhibition of cell proliferation and invasion, and angiogenesis (Figs. 4-6). Our results suggest that miR-451 acts as a tumor suppressor by down-regulating expression of IL6R gene (Figs. 2 and 3). We introduced miR-451 mimics into two types of cells, RKO and Hela, in which IL6R gene displays a relatively high expression. Interestingly, the IL6R mRNA was expressed a reduced level in both transfected cell lines compared to controls, suggesting that the mimics facilitate the cleavage and degradation of the IL6R mRNA (Fig. 2). For the siRNA studies, RNA oligonucleotides were directly introduced into cells instead of using vector transfection. It demonstrated a high efficiency of gene interference (Fig. 7). Moreover, the mimics for a variant of miR-451 was tested simultaneously, and we found that it does not target the IL6R gene (Figs. 2 and 3). The difference between wildtype and variant of miR-451 is only one base in the seed sequence (Table 1 ). It reflects that each base of the seed sequences of miRNAs can be critical for the specificity of the miRNAs. In our previous study, several variants of the miR-451 were identified [9]. Therefore it is possible that miR-451 family could target a broad range of genes.
IL6R is involved in IL6-STAT3-HIF signaling pathway, which plays a role in cell growth and carcinogenesis [17,18]. Badache et al. [19] suggested certain differences in the invasion and the proliferation pathways and that MAPK signaling is essential for IL6-induced invasion potential in breast cancer cells. Furthermore, the angiogenesis can be modulated by the IL6/STAT3 pathway [20]. IL6, originally identified as a B-cell differentiation factor, is a multifunctional cytokine that regulates the immune response, haemopoiesis, the acute phase response and inflammation. IL6 is produced by various types of cells and influences various cell types, and has multiple biological activities through its unique receptor system. IL6 exerts its biological activities through IL6R [21]. It can be inferred that the miR-451 may interfere with IL6-STAT3-HIF pathway to regulate cell growth and induce carcinogenesis, and influence MAPK pathway to increase invasive properties of cancer cells. Our study showed that angiogenesis was inhibited by miR-451 (Fig. 6). The mechanism behind this could be that the IL6/STAT3 pathway associated with angiogenesis is targeted by miR-451. Therefore, targeting these signaling circuits represents an effective therapeutic avenue for cancer prevention and treatment.

In conclusion, the IL6R was verified to be a target gene of the miR-451, which suppressed cell proliferation and invasion and angiogenesis, probably by down-regulating the expression of the IL6R gene. 

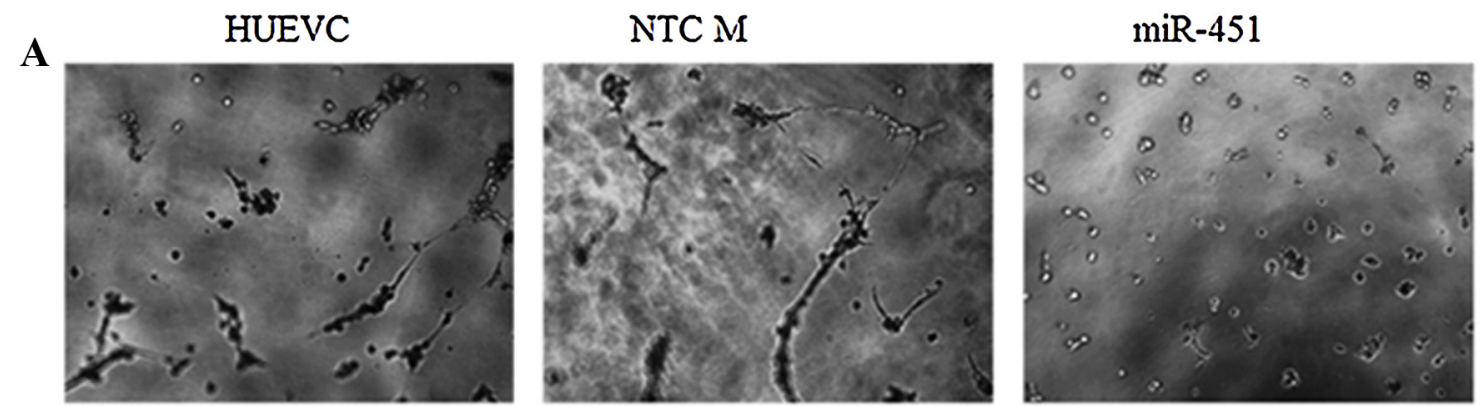

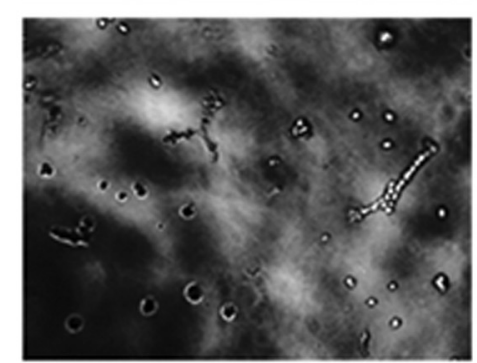

Variant

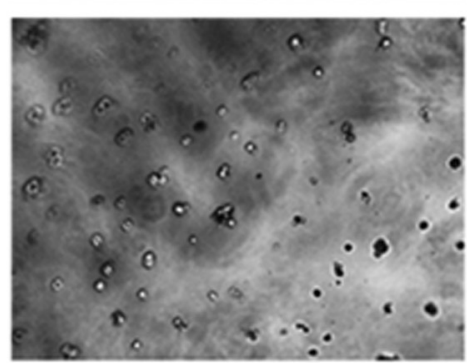

si-IL6R

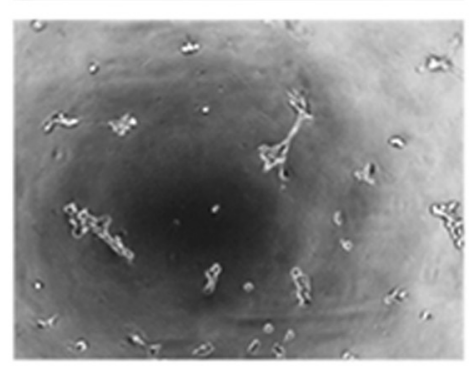

NTC S

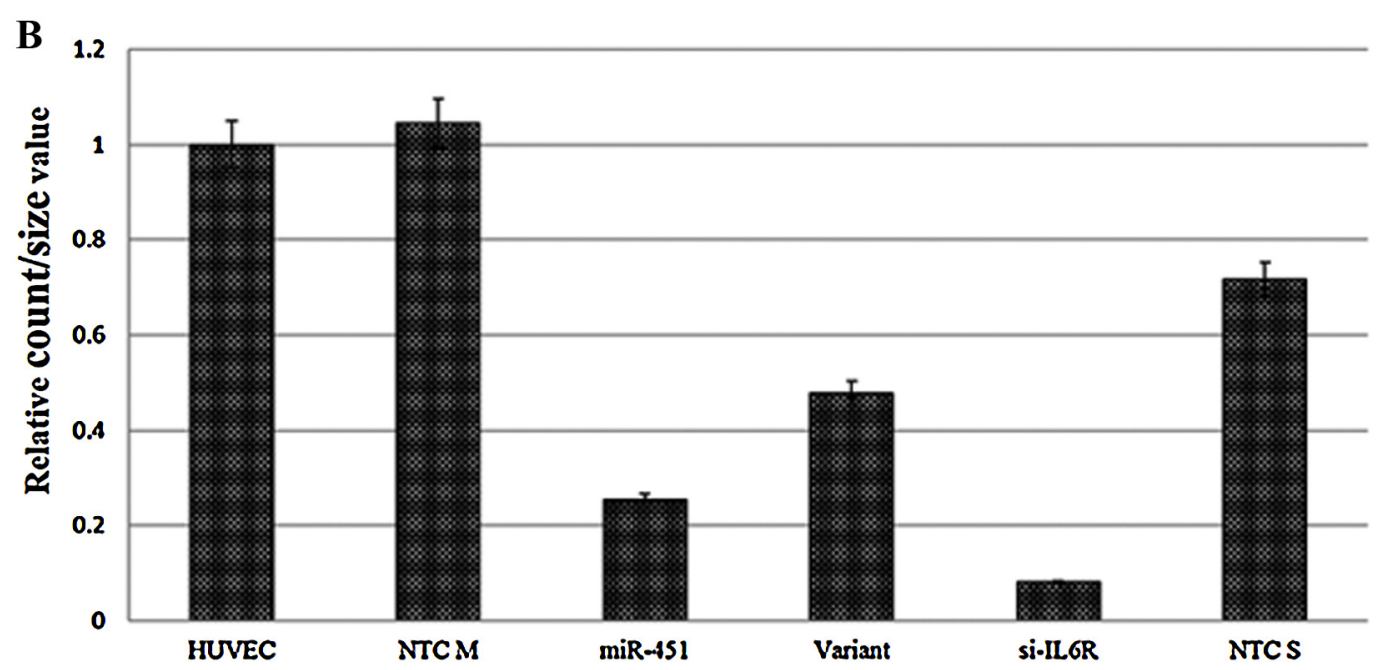

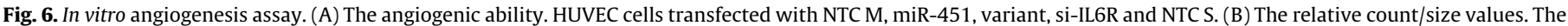

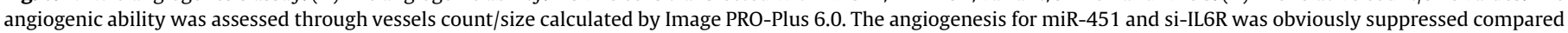
to controls $(P=0.006)$. (HUVEC, $1.00 \pm 0.0267 ;$ NTC M, 1.04 $\pm .0296 ;$ miR-451, $0.25 \pm 0.00658 ;$ Variant, $0.48 \pm 0.0152 ;$ si-IL6R, $0.08 \pm 0.00175 ;$ NTC S, $0.72 \pm 0.0178$ )

A
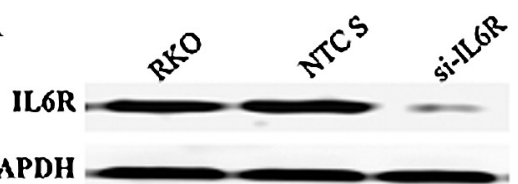

GAPDH

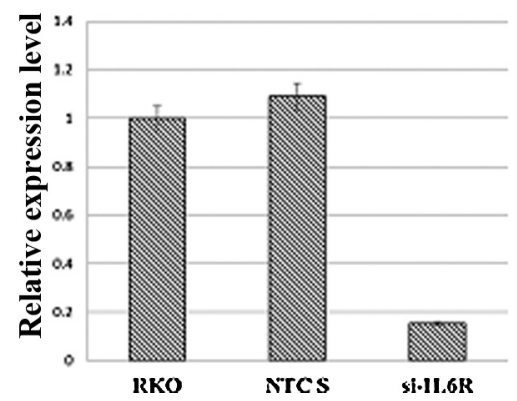

B

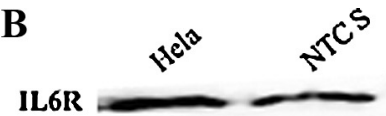

GAPDH

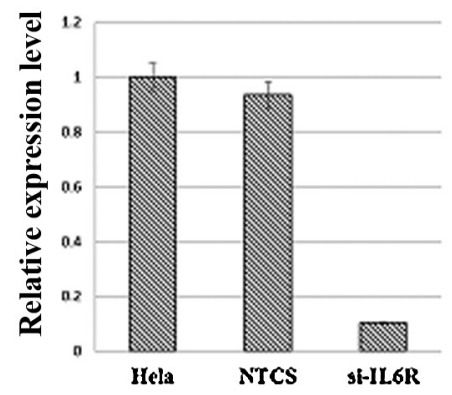

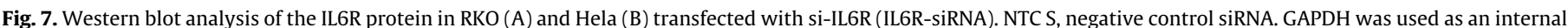

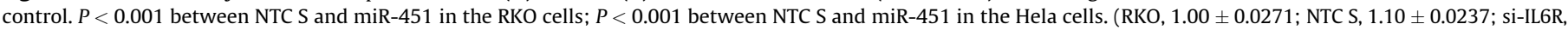
$0.15 \pm 0.00359$; Hela, $1.00 \pm 0.0271$; NTC S, $0.90 \pm 0.0234$; si-IL6R, $0.11 \pm 0.00227$ ). 


\section{Contributorship statement}

DL, HC: conception and design; DL, CL, XW: acquisition of data; DL, SI, HC: analysis and interpretation of data; DL, CL: drafting the article; HC: final approval of the version to be published.

\section{Conflict of interest}

The authors declare no conflicts of interest.

\section{References}

[1] Chu CY, Rana TM. Small RNAs: regulators and guardians of the genome. J Cell Physiol 2007;213:412-9.

[2] Lee RC, Feinbaum RL, Ambros V. The C. elegans heterochronic gene lin-4 encodes small RNAs with antisense complementarity to lin-14. Cell 1993;75:843-54.

[3] Lee Y, Kim M, Han J, Yeom KH, Lee S, Baek SH, et al. MicroRNA genes are transcribed by RNA polymerase II. EMBO J 2004;23:4051-60.

[4] Kim VN, Nam JW. Genomics of microRNA. Trends Genet 2006;22:165-73.

[5] Vatolin S, Navaratne K, Weil RJ. A novel method to detect functional microRNA targets. J Mol Biol 2006;358:983-96.

[6] Jannot G, Boisvert ME, Banville IH, Simard MJ. Two molecular features contribute to the Argonaute specificity for the microRNA and RNAi pathways in $C$. elegans. RNA 2008;14:829-35.

[7] Leung AK, Calabrese JM, Sharp PA. Quantitative analysis of Argonaute protein reveals microRNA-dependent localization to stress granules. Proc Natl Acad Sci USA 2006;103:18125-30.

[8] Tang Y, Liu D, Zhang L, Ingvarsson S, Chen H. Quantitative analysis of miRNA expression in seven human foetal and adult organs. PLoS ONE 2011;6:e28730.

[9] Liu D, Fan J, Mei M, Ingvarsson S, Chen H. Identification of miRNAs in a liver of a human fetus by a modified method. PLoS ONE 2009;26. 4:e7594.
[10] Liu D, Fan J, Zeng W, Zhou Y, Ingvarsson S, Chen H. Quantitative analysis of miRNA expression in several developmental stages of human livers. Hepatol Res 2010;40(August):813-22.

[11] Redova M, Poprach A, Nekvindova J, Iliev R, Radova L, Lakomy R, et al. Circulating miR-378 and miR-451 in serum are potential biomarkers for renal cell carcinoma. J Transl Med 2012;10:55

[12] Bitarte N, Bandres E, Boni V, Zarate R, Rodriguez J, Gonzalez-Huarriz M, et al MicroRNA-451 is involved in the self-renewal, tumorigenicity, and chemoresistance of colorectal cancer stem cells. Stem Cells 2011;29:1661-71.

[13] Namløs HM, Meza-Zepeda LA, Barøy T, Ostensen IH, Kresse SH, Kuijjer ML, et al. Modulation of the osteosarcoma expression phenotype by microRNAs. PLOS ONE 2012;7:e48086.

[14] Lau NC, Lim LP, Weinstein EG, Bartel DP. An abundant class of tiny RNAs with probable regulatory roles in Caenorhabditis elegans. Science 2001;294: 858-62.

[15] Brennecke J, Stark A, Russell RB, Cohen SM. Principles of microRNA-target recognition. PLoS Biol 2005;3:e85.

[16] Kim Y, Roh S, Lawler S, Friedman A. miR451 and AMPK mutual antagonism in glioma cell migration and proliferation: a mathematical model. PLoS ONE 2011;6:e28293.

[17] Krejci P, Prochazkova J, Bryja V, Jelinkova P, Pejchalova K, Kozubik A, et al Fibroblast growth factor inhibits interferon gamma-STAT1 and interleukin 6STAT3 signaling in chondrocytes. Cell Signal 2009;21:151-60.

[18] Anglesio MS, George J, Kulbe H, Friedlander M, Rischin D, Lemech C, et al. IL6STAT3-HIF signaling and therapeutic response to the angiogenesis inhibitor sunitinib in ovarian clear cell cancer. Clin Cancer Res 2011;17:2538-48.

[19] Badache A, Hynes NE. Interleukin 6 inhibits proliferation and, in cooperation with an epidermal growth factor receptor autocrine loop, increases migration of T47D breast cancer cells. Cancer Res 2001;61:383-91.

[20] Lamy S, Akla N, Ouanouki A, Lord-Dufour S, Béliveau R. Diet-derived polyphenols inhibit angiogenesis by modulating the interleukin-6/STAT3 pathway. Exp Cell Res 2012;318:1586-96.

[21] Mihara M, Hashizume M, Yoshida H, Suzuki M, Shiina M. IL-6/IL-6 receptor system and its role in physiological and pathological conditions. Clin Sci 2012;122:143-59. 